\title{
Influence of Movement Speed on Accuracy of Tracking Performance Following Stroke
}

\author{
HWA-KYUnG ShIn, PhD, PT ${ }^{1)}$, Ji-Won PARK, PhD, PT ${ }^{1)}$, Young UK RYU, PhD, $\mathrm{PT}^{1)}$ \\ 1) Department of Physical Therapy, College of Medical Science, Catholic University of Daegu: \\ 330 Geumrak 1-ri, Hayang-eup, Geongsan-si, Gyeongbuk, 712-702 Republic of Korea. \\ TEL:+82 53-850-3277, FAX: +82 53-850-3292,E-mail:ryu3091@cu.ac.kr
}

\begin{abstract}
Purpose] The present study was designed to examine the effects of movement speed on tracking accuracy of patients with stroke. [Subjects] Thirty stroke patients (16 patients with right hemiparesis and 14 patients with left hemiparesis, mean age $=56.2$ yrs, mean period after stroke $=19.2$ months) volunteered to participate in the study. [Subjects and Methods] Participants were required to track a continuous sinusoidal signal with rhythmic 3rd metacarpophalangeal (MCP) joint flexion-extension movements. The oscillation frequency of the signal was set at 0.2 $\mathrm{Hz}$ or $0.4 \mathrm{~Hz}$ with a total of 12 cycles per trial. The sinusoidal tracking signal and the participant's MCP motion signal were displayed overlapping together on a computer monitor. Participants performed 3 trials at each, $0.2 \mathrm{~Hz}$ and $0.4 \mathrm{~Hz}$, frequency condition. [Result] Tracking accuracy was significantly less accurate under the slower $0.2 \mathrm{~Hz}$ condition when tracking the tracking signal with the paretic finger. [Conclusion] Participants were unable to efficiently track the external signal with the paretic side, suggesting that motor execution on the paretic side was significantly impaired. Moreover, the decline in the execution of the tracking movement with decreasing movement frequency might result from a failure of the feedback system to frequently update the impaired finger's movements.
\end{abstract}

Key words: Speed-accuracy, Visuomotor tracking, Stroke

(This article was submitted Jul. 9, 2010, and was accepted Aug. 26, 2010)

\section{INTRODUCTION}

Coordination between a limb and an external event is an essential skill for the performance of many every day activities. For example, when driving a car along a road or aiming to follow a moving object, a precise relationship between the movements of the limbs and the continuously changing environmental condition is required to achieve the movement goal ${ }^{1)}$. To achieve the precise visuomotor tracking, normal functioning of the sensory and motor states is required.

A stroke or cerebrovascular accident (CVA) causes brain tissue damage which leads to focal deficits in the neurological system. Although the symptoms following a stroke depend on the area and amount of the brain that has been affected, patients with stroke have a variety of deficits in sensory, motor, perceptual, and language functions which are common factors impeding the restoration of functional activities after stroke ${ }^{2}$. Motor dysfunction is characterized by paralysis or weakness of the body on the opposite side from the brain lesion, and such motor dysfunction impairs performance of many daily activities. Stroke survivors also have a deficit in proprioception which plays an important role in monitoring the spatial orientation of limbs during movements ${ }^{3,4)}$. A decreased ability in the sensorimotor feedback loop, resulting from the impairment in proprioception, leads to delayed functional recovery, which in turn, negatively affects the quality of daily life ${ }^{5}$.

Joint tracking tasks have been used to elucidate the underlying mechanisms that enable the coordination of movements in response to external stimuli ${ }^{6}$. Tracking an external stimulus requires both sound motor ability and accurate cognitive information processing to produce a purposeful action in response to the stimulus. Thus, joint tracking tasks have been used as a tool to examine motor and cognitive abilities in various populations ${ }^{7-9}$. Also, joint tracking tasks can measure movement accuracy and error correction ability in response to movement speed. Chung et al. (2005) demonstrated that there were significant differences in tracking accuracies between healthy adults and patients with stroke during knee joint tracking with both a sound limb and an affected limb ${ }^{10)}$. Carey et al. (1998) reported that tracking accuracy in sound limbs of stroke patients was significantly decreased compared to healthy adults during a finger tracking task ${ }^{11)}$.

Proper proprioceptive input and muscle activation are required in order to accurately track an external stimulus ${ }^{12)}$. Thus, deterioration in tracking performance results from deficits in proprioception and/or muscle activation. Although many researchers have investigated tracking performance following stroke, the influence of movement speed on tracking accuracy has not received much attention. 
The present study was designed to examine the effects of movement speed on tracking accuracy while stroke patients attempted to track an external signal by flexing and extending a limb.

\section{SUBJECTS AND METHODS}

Thirty stroke patients (16 patients with right hemiparesis and 14 patients with left hemiparesis, mean age $=56.2 \mathrm{yrs}$, mean period after stroke $=19.2$ months) volunteered to participate in the study. The participation criteria for the present study were having suffered stroke for at least 1 year; a score of 24 to 30 on the Korean-mini mental state examination (K-MMSE); normal score in the motor-free visual perception test (MVPT); no recurrent stroke after the first attack; active range of motion (AROM) $>20^{\circ}$ of the metacarpophalangeal joint (MCP); be less than grade II on the modified Ashworth scale; no deformity of on the upper extremity (U/E), and no other neurological impairments. All participants voluntarily signed a consent form prior to the experiment.

Participants sat on a height-adjustable chair and faced a computer monitor. The participant's test arm was comfortably placed on a forearm plate in the prone position with $90^{\circ}$ flexion of the elbow and $10^{\circ}$ extension of the wrist, and the participant's forearm and wrist were secured with a Velcro ${ }^{\circledR}$. The motion of the 3rd MCP joint occur red in the sagittal plane. A double-axis electrogoniometer (Biometrics Ltd., Ladysmith) was attached to the table, and used to measure ROM in flexion and extension of the $3 \mathrm{rd}$ MCP joint. A mechanical joint in the double-axis electrogoniometer linked the proximal and distal arms: the proximal arm was placed on the longitudinal axis of the 3rd metacarpal bone and the distal arm was placed on the longitudinal axis of the 3 rd phalanx.

The double-axis electrogoniometer was calibrated to measure the motion of the 3rd MCP joint. The positional data of the joint was recorded by a MP $150^{\circledR}$ system (Biopac system Inc., Goleta, CA). The sinusoidal tracking signal produced by a personal computer was matched with the MCP ROM ranging from $10^{\circ}$ to $100^{\circ}$. The oscillation frequency of the signal was set at $0.2 \mathrm{~Hz}$ or $0.4 \mathrm{~Hz}$ with a total of 12 cycles in a trial. The sinusoidal tracking signal and the participant's MCP motion signal were displayed overlapping together on a computer monitor (Fig. 1). Participants were required to track the rhythmic 3rd MCP joint flexion-extension movements to the continuous sinusoidal signal. Participants performed 3 trials for each, $0.2 \mathrm{~Hz}$ and $0.4 \mathrm{~Hz}$, frequency condition.

Prior to data analysis, the positional data were filtered with a dual-pass Butterworth Filter with a cutoff frequency of $10 \mathrm{~Hz}$. The filtered data were used to compute the accuracy index (AI) described by Carey et al. (2002):

$$
\mathrm{AI}=100 *(\mathrm{P}-\mathrm{E}) / \mathrm{P}
$$

where $\mathrm{E}$ is the root mean square (RMS) error between the tracking signal and the participant's MCP motion signal, and $\mathrm{P}$ is the RMS difference between the tracking signal and

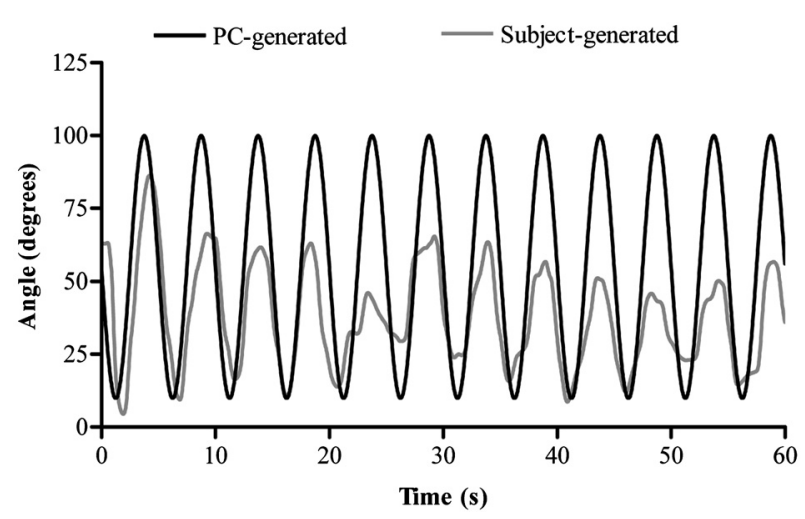

Fig. 1. Superimposition of the subject-generated wave on the PC-generated wave.

the midline separating the upper and lower portions of the tracking signal. The amplitude of $\mathrm{P}$ is the participant's range of MCP motion ${ }^{9}$. Thus, the AI is a normalized value of each participant's range of motion and ranges from $-100 \%$ to $100 \%$. The AI score becomes negative when the participant's MCP motion signal falls below the midline.

To test for tracking performance, the AI values were analyzed in a 2 Side (Paretic side, Nonparetic side) $\times 2$ Frequency $(0.2 \mathrm{~Hz}, 0.4 \mathrm{~Hz})$ ANOVA with repeated measures. SPSS (version 12.0) was used for statistical analysis. Simple main effect tests were performed to reveal differences when a significant interaction was present. The significance level was chosen as at $\alpha=0.05$.

\section{RESULTS}

The repeated measure ANOVA of the accuracy index (AI) revealed significant main effects of Side $(p<0.0001)$ and Frequency $(p<0.001)$. That is, the paretic limbs (mean $=68.44 \%)$ were significantly less accurate than the non-paretic limbs (mean $=80.74 \%)$, and the slower frequency condition $(0.2 \mathrm{~Hz}$, mean $=72.60 \%)$ was less accurate than the faster condition $(0.4 \mathrm{~Hz}$, mean $=76.58 \%)$. A significant interaction of Side $\times$ Frequency $(p<0.005)$ was found, and simple main effect tests revealed that the AI value was significantly smaller (less accurate) when performing the tracking task at $0.2 \mathrm{~Hz}$ (mean=65.12\%) than at $0.4 \mathrm{~Hz}$ (mean=71.82\%) when participants tracked with the paretic side.

\section{DISCUSSION}

The main purpose of the present study was to investigate the effects of movement speed on tracking accuracy of patients with stroke. We demonstrated that the tracking accuracy was significantly less accurate under the slower $0.2 \mathrm{~Hz}$ condition when tracking target waveforms with the paretic finger. When tracking with the nonparetic finger, however, there was no significant effect of movement speed on tracking accuracy. Thus, movement speed influenced accuracy differentially between the paretic and nonparetic fingers. 
In an aiming task, Fitts' law predicts a linear relationship between movement time and spatial accuracy, because the human motor control system requires more time to move effectors for spatial accuracy ${ }^{13)}$. However, if more than enough time to acquire spatial accuracy is provided in an aiming task, there is a time beyond which the spatial accuracy does not increase any further. It is reasonable to interpret that the tracking frequencies $(0.2$ and $0.4 \mathrm{~Hz})$ used in this study were too slow to cause differences in spatial accuracy in the nonparetic fingers. This suggests that when tracking with the nonparetic fingers the visuomotor system could resolve two frequencies without any perceptual or motor difficulty.

Previous tracking studies using the forearm as an effector to track an external signal have used tracking frequencies from 0.6 to $2.8 \mathrm{~Hz}$ (e.g., $0.67 \mathrm{~Hz}$ in Ceux et al., 2003, $0.8 \mathrm{~Hz}$ in Ryu and Buchanan, 2009, $1 \mathrm{~Hz}$ in Buekers et al., 2000, 1 $\mathrm{Hz}$ to $2.8 \mathrm{~Hz}$ in Peper and Beek, 1998) for neurologically healthy adults ${ }^{1,14,15)}$. In those studies, differential stability became obvious around $0.6 \mathrm{~Hz}$ as in-phase tracking $\left(0^{\circ}\right.$ difference between the external signal and limb's motion) was more accurate and stable than anti-phase tracking $\left(180^{\circ}\right.$ phase difference). These findings suggest that regardless of tracking patterns the visuomotor system of healthy adults can produce stable and accurate tracking performance below $0.6 \mathrm{~Hz}$. However, for chronic stroke patients, tracking at even lower frequencies could be a challenge for paretic limbs. Carey and colleagues asked stroke patients to track waveforms using frequency conditions as low as $0.1 \mathrm{~Hz}^{9}$. They demonstrated that tracking performance measured by spatial accuracy was impaired in paretic fingers at the slower tracking frequencies. However, the impact of movement frequency on spatial accuracy did not receive further investigation in their researches.

According to our literature searches, not many studies have examined the relationship between movement speed and accuracy in visuomotor tracking tasks performed by patients with stroke. Although Patten et al. (2003) did not give further attention to this issue, they reported the results of tracking accuracy by tracking speed for a patient with chronic stroke ${ }^{6}$. They demonstrated that when tracking a sinusoidal wave with the paretic elbow the tracking accuracy was less accurate at the slow arm speed $(25 \%$, approximately $0.08 \mathrm{~Hz})$ than at the medium speed $(45 \%$, approximately $0.15 \mathrm{~Hz}$ ). Although Patten et al. utilized slower tracking frequencies and a different effector (forearm) to track the sinusoidal signal, compared to the current study, their main finding was consistent with the current findings. However, these findings from the visuomotor tracking tasks are not consistent with Fitt's law which predicts an increased spatial accuracy with slower movement speed, the so-called speed-accuracy trade-off. Why did tracking accuracy degrade at the slower frequency when tracking with the paretic finger in the current visuomotor tracking task? One possible explanation may be the nature of the task. The visuomotor tracking task used in the present study requires continuous accuracy constraints for participants during movements of their fingers to enable continuous and precise finger control as well as visual feedback to track the external signal. Visuomotor tracking studies have demonstrated that a slower movement frequency causes an increased non-smoothness of movement producing directional changes in the velocity profile of the trajectory. The degree of the non-smoothness of the movement is dependent on the utilization of feedback information that becomes salient when a closed-loop feedback control is required to track an external signal ${ }^{16-18)}$. During increased closed-loop control, motor control systems require frequent updating of the limb's movements, acting to external events, in order to reduce tracking error ${ }^{17)}$.

In the present study, the slower $0.2 \mathrm{~Hz}$ condition required increased closed-loop control with frequent updating of the limb's movements. Importantly, patients with stroke have impaired proprioception that results in decreased ability in the sensorimotor feedback loops ${ }^{3)}$. Taken together, it is reasonable to expect that tracking performance with a paretic limb would be degraded at the $0.2 \mathrm{~Hz}$ frequency which required increased (frequent) feedback control of the finger's movements. This increased closed-loop control at the slower $0.2 \mathrm{~Hz}$ frequency impacted the impaired finger control more than at the $0.4 \mathrm{~Hz}$ frequency.

In conclusion, the present study demonstrated that stroke patients were unable to efficiently track an external signal with a paretic limb, suggesting that motor execution is significantly impaired in stroke patients. Moreover, the decline in the execution of the tracking movement with decreasing movement frequency might result from a failure of the feedback system to frequently update the impaired finger's movements.

\section{REFERENCES}

1) Ryu YU, Buchanan JJ: Learning an environment-actor coordination skill: Visuomotor transformation and coherency of perceptual structure. Exp Brain Res, 2009,196: 279-293.

2) O'Sullivan SB, Schmitz TJ: Physical rehabilitation 5th edition. FA Davis Co, 2006.

3) Shin HK, Cho SH, Jeon HS et al.: Cortical effect and functional recovery by the electromyography-triggered neuromuscular stimulation in chronic stroke patients. Neurosci Lett, 2008, 442: 174-179.

4) Cho SH, Shin HK, Kwon YH et al.: Cortical activation changes induced by visual biofeedback tracking training in chronic stroke patients. NeuroRehabilitation, 2007, 22: 77-84.

5) Tyson SF, Hanley M, Chillala J et al.: Sensory loss in hospital-admitted people with stroke: Characteristics, associated factors, and relationship with function. Neurorehabil Neural Repair, 2008, 22: 166-172.

6) Patten C, Kothari D, Whitney J et al.: Reliability and responsiveness of elbow trajectory tracking in chronic poststroke hemiparesis. J Rehabil Res Dev, 2003, 40: 487-500.

7) Carey JR, Anderson KM, Kimberley TJ et al.: FMRI analysis of ankle movement tracking training in subject with stroke. Exp Brain Res, 2004, 154: 281-290.

8) Carey JR, Comnik KT, Ingman MS.: Finger movement tracking control in 8 and 9 year old males and females in stimulus response compatible and noncompatible hand positions. Cortex, 2001, 37: 433-439.

9) Carey JR, Kimberley TJ, Lewis SM et al.: Analysis of fMRI and finger tracking training in subjects with chronic stroke. Brain, 2002, 125 (Pt 4): 773 788.

10) Chung YJ, Cho SH, Lee YH: Effect of the knee joint tracking training in closed kinetic chain condition for stroke patients. Restor Neurol Neurosci, 2006, 24: 173-180.

11) Carey JR, Baxter TL, Di Fabio RP: Tracking control in the nonparetic hand of subjects with stroke. Arch Phys Med Rehabil, 1998, 79: 435-441.

12) Sainburg RL, Poizner H, Ghez C: Loss of proprioception produces deficits in interjoint coordination. J Neurophysiol, 1993, 70: 2136-2147. 
13) Fitts PM: The information capacity of the human motor system in controlling the amplitude of movement. J Exp Psychol, 1954, 47: 381-391.

14) Ceux T, Buekers MJ, Montagne G: The effects of enhanced visual feedback on human synchronization. Neurosci Lett, 2003, 349: 103-106.

15) Buekers MJ, Bogaerts HP, Swinnen SP et al.: The synchronization of human arm movements to external events. Neurosci Lett, 2000, 290: 181-184.
16) Craik KJ: Theory of the human operator in control systems; the operator as an engineering system. Br J Psychol Gen Sect, 1947, 38 (Pt 2): 56-61.

17) Miall RC, Weir DJ, Stein JF: Intermittency in human manual tracking tasks. J Mot Behav, 1993, 25: 53-63.

18) Russell DM, Sternad D: Sinusoidal visuomotor tracking: Intermittent servocontrol or coupled oscillations? J Mot Behav, 2001, 33: 329-349. 